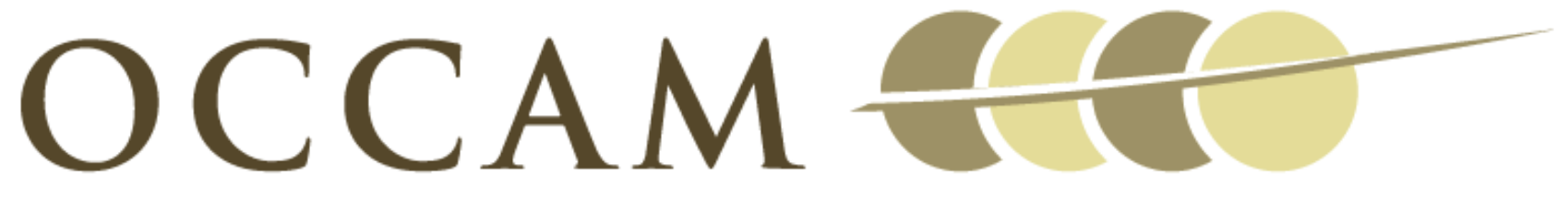

OXFORD CENTRE FOR COLLABORATIVE APPLIED MATHEMATICS

Report Number 09/28

Quasi-steady state reduction of molecular motor-based models of directed intermittent search

by

Jay M. Newby and Paul C. Bressloff

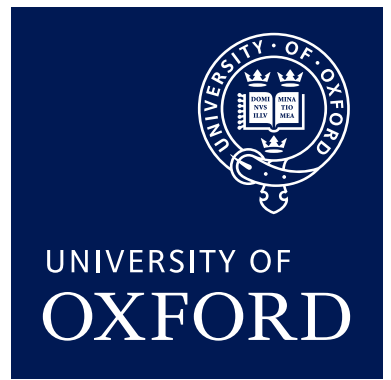

Oxford Centre for Collaborative Applied Mathematics Mathematical Institute 24 - 29 St Giles'

Oxford

OX1 3LB

England 



\title{
Quasi-steady state reduction of molecular motor-based models of directed intermittent search
}

\author{
Jay M. Newby ${ }^{1}$ and Paul C. Bressloff ${ }^{1,2}$ \\ 1 Department of Mathematics, University of Utah, Salt Lake City, UT 84112 USA \\ 2 Mathematical Institute, University of Oxford, 24-29 St. Giles', Oxford OX1 \\ $3 \mathrm{LB}, \mathrm{UK}$
}

The date of receipt and acceptance will be inserted by the editor

\begin{abstract}
We present a quasi-steady state reduction of a linear reactionhyperbolic master equation describing the directed intermittent search for a hidden target by a motor-driven particle moving on a one-dimensional filament track. The particle is injected at one end of the track and randomly switches between stationary search phases and mobile, non-search phases that are biased in the anterograde direction. There is a finite possibility that the particle fails to find the target due to an absorbing boundary at the other end of the track. Such a scenario is exemplified by the motordriven transport of vesicular cargo to synaptic targets located on the axon or dendrites of a neuron. The reduced model is described by a scalar FokkerPlanck (FP) equation, which has an additional inhomogeneous decay term that takes into account absorption by the target. The FP equation is used to compute the probability of finding the hidden target (hitting probability) and the corresponding conditional mean first passage time (MFPT) in terms of the effective drift velocity $V$, diffusivity $D$ and target absorption rate $\lambda$ of the random search. The quasi-steady state reduction determines $V, D$ and $\lambda$ in terms of the various biophysical parameters of the underlying motor transport model. We first apply our analysis to a simple 3-state model and show that our quasi-steady state reduction yields results that are in excellent agreement with Monte Carlo simulations of the full system under physiologically reasonable conditions. We then consider a more complex multiple motor model of bidirectional transport, in which opposing motors compete in a "tug-of-war," and use this to explore how ATP concentration might regulate the delivery of cargo to synaptic targets.
\end{abstract}

Key words Intracellular transport, molecular motors, random search, quasi-steady state, axons, dendrites 


\section{Introduction}

The neuron is one of the most complex animal cell types. Due to its size, shape and function, the trafficking and targeting of vital resources such as proteins and lipid membrane throughout a neuron is an extremely challenging problem $[22,3]$. Each neuron has a cell body or soma, which is roughly spherical in shape and contains the nucleus where transcription of mRNA occurs. The plasma membrane of the neuron extends out to form several tubular protrusions corresponding to a single axon and one or more dendrites. The axon is the largest in diameter and typically extends further, whereas the dendrites tend to be more extensively branched. Both the axon and dendrites contain highly regulated, protein-rich subcellular compartments that constitute synaptic contacts between neurons. In order to generate new synaptic contacts during synaptogenesis or to maintain and modify existing synapses in response to synaptic activity from other neurons, it is necessary to synthesize new protein products and localize them at the appropriate synaptic site. This can be achieved either by globally transporting newly synthesized proteins from the soma, which tends to occur during synaptogenesis [42], or by transporting newly transcribed mRNA from the soma followed by local protein synthesis $[21,39]$. In either case, resources must be transported long distances and delivered to specific synaptic locations at specific times. Moreover, a breakdown in the processes responsible for the transport and localization of resources has been implicated in a variety of neurological disorders.

The long distances between the soma and distal synapses on the axon or dendrites necessitates a faster means of transportation than passive transport. Diffusion inside the cytosol or along the plasma membrane is a means by which dissolved macromolecules can be passively transported without any input of energy. There are two main problems with passive diffusive transport in neurons. First, it takes far too long to travel the long distances necessary to reach targets within an axon or dendrite. Second, diffusive motion is unbiased, making it difficult to sort resources to specific areas within a neuron. Active intracellular transport can overcome these difficulties so that movement is both faster and biased in a certain direction, but does so at a price. Active transport cannot occur under thermodynamic equilibrium, which means that energy must be consumed by this process. The type of active transport that we consider in this paper uses molecular motors to carry resources along microtubular filament tracks. Microtubules are polarized polymeric filaments with biophysically distinct $(+)$ and $(-)$ ends, and this polarity determines the preferred direction in which an individual molecular motor moves. For example, kinesin moves towards the $(+)$ end whereas dynein moves towards the $(-)$ end $[20,40]$. Each motor protein will undergo a sequence of conformational changes after reacting with one or more ATP molecules, causing it to step forward along the microtubule in its preferred direction. Thus, ATP provides the energy necessary for the molecular motor to do work in the form of pulling its cargo along the microtubule in a 
biased direction. Motor driven transport along microtubules is responsible for the distribution of many components necessary for the proper function of a neuron [19]. Molecular motors, such as kinesin and dynein, have been implicated in the transport of neurotransmitter receptors [43], mitochondria [30], ER sub-compartments [2], neurofilaments [11], ribosomes, and mRNA $[37,13]$. Transport in the axon has been implicated in many neurological diseases such as Alzheimer's, Parkinson's, and Huntington's disease [38,12, 28].

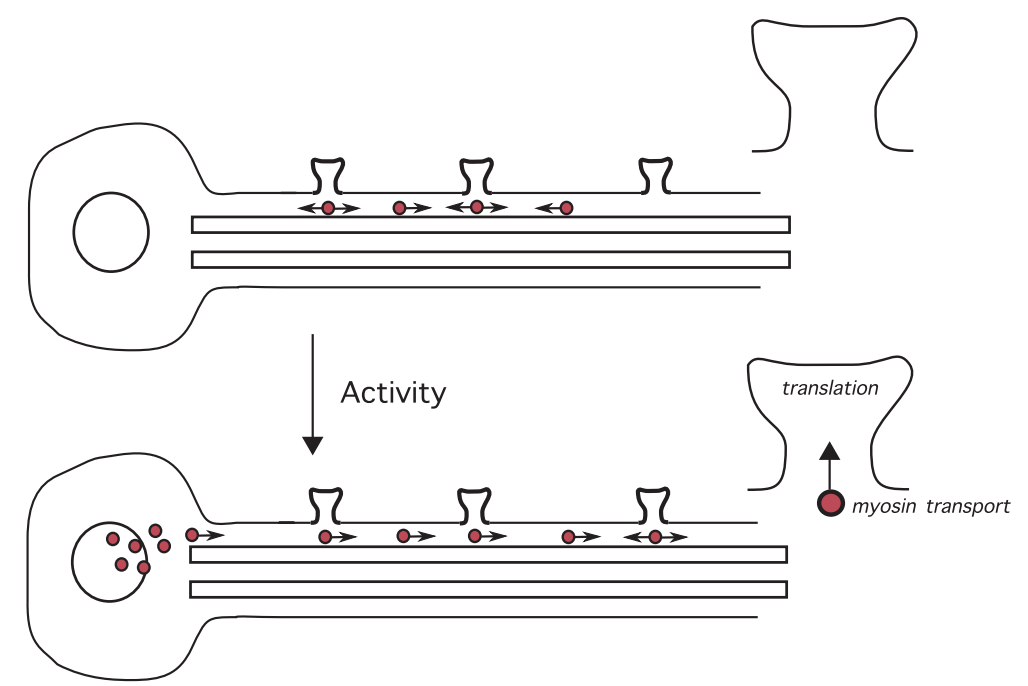

Fig. 1 Schematic diagram illustrating mRNA granule mobility in dendrites. Under basal conditions most granules are either stationary (or exhibit localized oscillations), whereas a minority exhibit bidirectional transport. $\mathrm{KCl}$ depolarization activates transcription of mRNA at the cell body and converts existing stationary granules into anterograde granules [37].

In experiments where fluorescent labeling and live-cell imaging have been used to track the position of a cargo, the movement along a dendrite or axon is seen to randomly pause and switch direction [37,13,32]. Moreover, the probability of being in a particular motile state can be modified by activity. All of these features occur in an emerging model of mRNA transport and localization within dendrites $[21,8]$, which is illustrated schematically in Fig. 1. Newly transcribed mRNA within the nucleus binds to proteins that inhibit translation, thus allowing the mRNA to be sequestered away from the protein-synthetic machinery within the cell body. The repressed mRNAs are then packaged into ribonucleoprotein granules that are subsequently transported into the dendrite via kinesin and dynein motors along microtubules. Finally, the mRNA is localized to an activated synapse by actin-based myosin motor proteins, and local translation is initiated following neutralization of the repressive mRNA-binding protein. It has also been 
found that under basal conditions the majority of granules in dendrites are stationary or exhibit small oscillations around a few synaptic sites. However, other granules exhibit rapid retrograde (towards the cell body) or anterograde (away from the cell body) motion consistent with bidirectional transport along microtubules. Following depolarization of the cell, there is an enhancement of dendritically localized mRNA due to a combination of newly transcribed granules being transported into the dendrite, and the conversion of some stationary or oscillatory granules already present in the dendrite into anterograde-moving granules [37].

The random switching between different motile states can be explained using a biophysical model of the cargo and microtubule interacting via multiple molecular motors [44]. The motors interact through the forces they each place on the cargo. If the set of motors transporting a cargo is comprised of motors with opposing directional preference then they may compete in a tug-of-war [44,31], but see [23]. Movement of the cargo is then ultimately determined by the random binding and unbinding of the motors to the microtubule. The unbinding rate depends on the force applied to the motor. If a force is applied opposite to the preferred direction of a motor, then it is more likely to unbind from the microtubule. We can consider all of the motors attached to a cargo as a motor complex so that the different states of the motor complex represent different configurations of bound and unbound motors. This biophysical model explains how a cargo is transported by molecular motors, but it can't explain how that cargo is delivered to the correct location. In order for a motor complex to correctly localize its cargo to a specific site or target it must be released from its motor in a controlled manner. Some of the molecular mechanisms that cause a cargo to detach from a molecular motor have been identified [17]. In most cases, a protein dissolved in the cytosol reacts with an adaptor protein that binds a cargo to the motor, causing the cargo to be released. However, when a cargo is pulled at a relatively high velocity it doesn't have much time to explore local space and is therefore much less likely to participate in such a reaction. Therefore, one possible interpretation of the frequent pauses observed during motor transport is that it provides a mechanism to improve the reaction kinetics required to localize the cargo to its target by giving it more time to explore local space. Such a hypothesis is consistent with the observation that stationary mRNA granules tend to be colocalized with synaptic sites [37]. This then leads to a simple model of cargo delivery in which there are transitions of the internal state of the motor complex between directed movement states and stationary searching states. If the transitions between these states are governed by chemical reactions under the influence of thermodynamic fluctuations, then the model becomes a random search.

Random search has recently been used to model a wide range of problems, including the behavior of foraging animals $[4,41,5]$, the active transport of reactive chemicals in cells [27] and DNA binding kinetics [7,18]. One particular class of models treats a random searcher as a particle that switches between a slow motion (diffusive) or stationary phase in which tar- 
get detection can occur and a fast motion 'ballistic' phase; transitions between bulk movement states and searching states are governed by a Markov process $[5,6,27]$. Under the assumptions that the random search is unbiased and that the probability of finding a single hidden target is unity, it can be shown that there exists an optimal search strategy given by the durations of each phase that minimize the mean search time to find the target. Motivated by experimental observations of the motor-driven transport of mRNA granules [37,13], see Fig. 1, we recently extended a one-dimensional version of these models to the case of a directed intermittent search process, in which the motion is biased and there is a non-zero probability of failing to find the target (due to competition with other targets or degradation) [9]. In particular, we considered a 3 -state model consisting of two moving states (anterograde and retrograde) and one stationary search state. This provides a simple model for the motor-driven bidirectional transport of cargo from the soma to synaptic targets along dendrites and axons. The associated master equation takes the form of a linear reaction-hyperbolic equation, which can be used to analyze the efficiency of the random search by calculating the hitting probability and conditional mean first passage time (MFPT) for finding the target [9]. However, our 3-state model makes a number of simplifying assumptions. In particular, it ignores the action of multiple motors highlighted above, which would lead to a higher number of internal velocity states, and it does not take into account the branching structure of axons and dendrites. Unfortunately, the analysis of the underlying master equation becomes considerably more difficult as the complexity of the motor transport model increases.

In this paper we carry out a quasi-steady state reduction of a linear reaction-hyperbolic equation corresponding to a general master equation for directed intermittent search. The reduced model is described by a onedimensional Fokker-Planck equation, which can then be used to analyze random search in biophysically realistic motor transport models. Our reduction is based on the observation that the state transition rates of the molecular motor complex are fast compared to the characteristic velocities. A number of authors have analyzed linear reaction-hyperbolic equations in this regime but have focused on the wave-like properties of the transport process rather than the delivery of cargo to hidden targets $[36,14,15,10]$. For example, Reed et. al. [36] used singular perturbation theory to show that the transport of a chemical along an axon can be analyzed in terms of an approximate traveling-wave solution of a scalar advection diffusion equation for the chemical concentration. Subsequent work rigorously established the validity of this scalar reduction for a wide range of linear reactionhyperbolic equations $[14,15,10]$. In models of directed intermittent search, approximate traveling wave solutions no longer exist due to the presence of a target. Nevertheless, it is still possible to reduce the master equation to a corresponding scalar Fokker-Planck equation, which has an additional inhomogeneous decay term that takes into account absorption by the target. After introducing the 3-state model in section 2, we use a projection 
method along the lines of Gardiner [16] to carry out a quasi-steady state reduction of both the 3-state model and a more general multi-state model (section 3). We then use the resulting Fokker-Planck equation to calculate the hitting probability and MFPT for finding the target, and show that our results are in excellent agreement with Monte Carlo simulations of the 3 -state system under physiologically reasonable choices for the parameters (section 4). Finally, we apply the general quasi-steady state reduction to a more complex multiple motor model, and use this to explore how ATP concentration might regulate the delivery of cargo to synaptic targets (section $5)$.

\section{Stochastic model of motor-driven transport}

A simple stochastic model of the directed intermittent search for a hidden (synaptic) target by a motor-driven particle was previously analyzed by the authors [9]. Consider a single particle moving along a one-dimensional track of length $L$, see Fig. 2. Such a track could represent a single microtubular filament. For the moment, we neglect the fact that there are multiple filaments running along the length of axon or dendrite and that motors can switch between them (but see section 5). For simplicity, we will assume throughout that a particle is initially injected at one end of the track, which we take to be at $x=0$. This would correspond to the case of a particle entering a primary dendrite from the soma, for example. (However, one could also consider a particle injected into the interior of the track, which would correspond to the case of a stationary particle being recruited from a local intracellular pool following depolarization of the cell, see Fig. 1). Within the interior of the track, $0<x<L$, the particle is taken to be in one of three states labeled by $n=0, \pm$ : stationary $(n=0)$, moving to the right (anterograde) with speed $v(n=+)$, or moving to the left (retrograde) with speed $-v(n=-)$. Transitions between the three states are governed by a discrete Markov process. We further assume that there is a hidden (synaptic) target at a fixed but unknown location $x=X$. If the particle is within a distance $l$ of the target and is in the stationary state, then the particle can detect or, equivalently, be absorbed by the target at a rate $\kappa$. We assume throughout that $l<X$ and $l<L-X$ so the domain of detectability lies fully within the interval $[0, L]$.

Let $Z(t)$ and $N(t)$ denote the random position and state of the particle at time $t$ and define $\mathbb{P}(x, t, n \mid y, 0, m) d x$ as the joint probability that $x \leq$ $Z(t)<x+d x$ and $N(t)=n$ given that initially the particle was at position $Z(0)=y$ and was in state $N(0)=m$. Setting

$$
p_{n}(x, t) \equiv \sum_{m} \mathbb{P}(x, t, n \mid 0,0, m) \sigma_{m}
$$

with initial condition $p_{n}(x, 0)=\delta(x) \sigma_{n}, \sum_{m=1}^{n} \sigma_{m}=1$, we have the following master equation describing the evolution of the probability densities for 
$t>0$ :

$$
\begin{aligned}
\partial_{t} p_{+} & =-v \partial_{x} p_{+}-\beta_{+} p_{+}+\alpha p_{0} \\
\partial_{t} p_{-} & =v \partial_{x} p_{-}-\beta_{-} p_{-}+\alpha p_{0} \\
\partial_{t} p_{0} & =\beta_{+} p_{+}+\beta_{-} p_{-}-2 \alpha p_{0}-\kappa \chi([x-X] / l) p_{0}
\end{aligned}
$$

with

$$
\chi(x)=\left\{\begin{array}{l}
1, \text { if }|x|<1 \\
0, \text { otherwise }
\end{array}\right.
$$

Here $\alpha, \beta_{ \pm}$are the transition rates between the stationary and mobile states as indicated in Fig. 2. The master equation (2.2) is supplemented by a reflecting boundary condition at $x=0$ and an absorbing boundary condition at $x=L$. That is,

$$
p_{-}(0, t)=p_{+}(0, t), \quad p_{-}(L, t)=0 .
$$

The absorbing boundary takes into account the fact that transported cargo can be degraded or absorbed by other targets downstream to the given target. Finally, we assume that the transport is partially biased in the anterograde direction by taking $\beta_{+}<\beta_{-}$, which implies that the particle spends more time in the anterograde state than the retrograde state. Unidirectional transport is obtained in the limit $\beta_{-} \rightarrow \infty$.

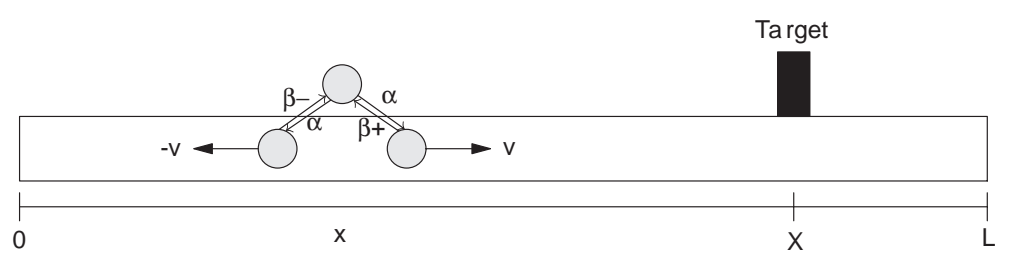

Fig. 2 Schematic diagram illustrating a model of a motor-driven particle moving along a one-dimensional track of length $\mathrm{L}$. The particle can transition from a moving state with velocity $\pm v$ at a rate $\beta_{ \pm}$and from a stationary searching state at a rate $\alpha$. A target of width $2 l$ is located at a fixed but unknown location $x=X$.

In our previous work [9], we calculated two important quantities characterizing the efficacy of the directed intermittent search process. The first is the hitting probability $\Pi$ that a particle starting at $x=0$ at time $t=0$ finds the target, that is, the particle is absorbed somewhere within the domain $X-l \leq x \leq X+l$ rather than at the boundary $x=L$. The second is the conditional mean first passage time (MFPT) $T$, which is the mean time it takes for the particle to find the target given that it is eventually absorbed by the target. Clearly it would be advantageous for the particle to minimize the search time $T$ and maximize the hitting probability $\Pi$. However, these two requirements compete with each other so that, in contrast to other studies of intermittent search for which $\Pi=1$ and the motion 
is unbiased [5,6,27], there is not a single optimal strategy [9]. This can be seen heuristically in the case of unidirectional transport where the particle is either stationary or undergoes anterograde motion. Such a particle can reach the target more quickly by having a higher probability of being in the mobile state. However, this also increases the chance of overshooting the target without detecting it and thus reduces the hitting probability. In cases where there are multiple particles being actively transported along an axon or dendrite, it could be argued that the only important factor is minimizing the MFPT irrespective of the hitting probability. However, in general there will be multiple targets competing for resources. Moreover, the extensive branching of axons and dendrites could hinder the delivery of particles to distant sites. Therefore, it is important to keep track of both the hitting probability and the MFPT. Unfortunately, the calculation of $\Pi$ and $T$ becomes considerably more difficult when the complexity of the motor transport model increases or when branching structures are included. Therefore, in this paper we carry out a quasi-steady state reduction of the master equation (2.2) to a one-dimensional Fokker-Planck equation and use the latter to calculate $\Pi$ and $T$. Such a reduction can then be applied to more complex models (see section 5).

\section{Quasi-steady state reduction}

In the absence of an absorbing target $(\kappa \equiv 0)$, the master equation $(2.2)$ belongs to a general class of linear reaction-hyperbolic equations studied previously by a number of groups $[36,14,15,10]$. Under the assumption that the transition rates are sufficiently fast, Reed et. al. [36] used singular perturbation methods to carry out an asymptotic expansion of a solution whose leading order term is given by an approximate traveling wave solution of a corresponding one-dimensional advection-diffusion equation (with the state variables $p_{m}$ interpreted as concentrations rather than probabilities). They then showed how such a solution matches wave-like behavior observed experimentally in the axonal transport of certain chemicals. The validity of this reduction was subsequently proved rigorously under a wide range of conditions in $[14,15,10]$. The full system (2.2) does not support approximate traveling wave solutions due to the presence of the target inhomogeneity. Nevertheless, it is still possible to reduce the master equation to a corresponding one-dimensional Fokker-Planck equation (under the probabilistic interpretation of the state variables $p_{m}$ ) in the case of fast transition rates. In this section we carry out a quasi-steady state reduction of the 3-state model using a projection method (see for example [16]) and then show how to extend the construction to a more general class of multi-state models. 


\subsection{Reduction of 3-state model}

We first non-dimensionalize the master equation (2.2) by rescaling space and time according to

$$
x \rightarrow \frac{x}{l}, \quad t \rightarrow t \frac{v}{l},
$$

where $l=1 \mu m$ is the typical size of the target. Assuming that the transition rates $\alpha, \beta_{ \pm}$are large compared to $v / l$, we introduce the dimensionless parameters $a=\epsilon \alpha l / v$ and $b_{ \pm}=\epsilon \beta_{ \pm} l / v$ where $\epsilon \ll 1$. The master equation (2.2) then becomes

$$
\begin{aligned}
\partial_{t} p_{+} & =\frac{1}{\epsilon}\left(-b_{+} p_{+}+a p_{0}\right)-\partial_{x} p_{+} \\
\partial_{t} p_{-} & =\frac{1}{\epsilon}\left(-b_{-} p_{-}+a p_{0}\right)+\partial_{x} p_{-} \\
\partial_{t} p_{0} & =\frac{1}{\epsilon}\left(b_{+} p_{+}+b_{-} p_{-}-2 a p_{0}\right)-k(x) p_{0},
\end{aligned}
$$

where

$$
k(x)=k_{0} \chi(x-X), \quad k_{0}=\kappa l / v .
$$

Note that the target location $X$ and track length $L$ are now taken to be in units of the target half-width $l$. The master equation can be rewritten in the matrix form

$$
\partial_{t} \mathbf{p}=\frac{1}{\epsilon} A \mathbf{p}+\mathcal{L}(\mathbf{p}),
$$

where $p=\left(p_{+}, p_{-}, p_{0}\right)^{T}, A$ is the matrix

$$
A=\left[\begin{array}{ccc}
-b_{+} & 0 & a \\
0 & -b_{-} & a \\
b_{+} & b_{-} & -2 a
\end{array}\right]
$$

and $\mathcal{L}$ is the linear operator

$$
\mathcal{L}(\mathbf{f})=\left[\begin{array}{c}
-\partial_{x} f_{1} \\
\partial_{x} f_{2} \\
-k(x) f_{3}
\end{array}\right] .
$$

The left nullspace of the matrix $A$ is spanned by the vector

$$
\psi=\left(\begin{array}{l}
1 \\
1 \\
1
\end{array}\right)
$$

and the right nullspace is spanned by

$$
\mathbf{p}^{s s}=\frac{1}{\gamma}\left(\begin{array}{c}
\frac{1}{b_{+}} \\
\frac{1}{b_{-}} \\
\frac{1}{a}
\end{array}\right) .
$$


The normalization factor $\gamma$ is chosen so that $\psi^{T} \mathbf{p}^{s s}=1$, that is,

$$
\gamma=\frac{1}{b_{+}}+\frac{1}{b_{-}}+\frac{1}{a} .
$$

Let $u=\psi^{T} \mathbf{p}$ and $\mathbf{w}=\mathbf{p}-u \mathbf{p}^{s s}$ such that $\psi^{T} \mathbf{w}=0$. We can interpret $u$ as the component of $\mathbf{p}$ in the left nullspace of $A$, whereas $\mathbf{w}$ is in the orthogonal complement.

Multiplying both sides of (3.2) by $\psi^{T}$ we obtain

$$
\partial_{t} u=\psi^{T} \mathcal{L}\left(u \mathbf{p}^{s s}+\mathbf{w}\right) .
$$

Substituting $\mathbf{p}=\mathbf{w}+u \mathbf{p}^{s s}$ into (3.2) yields

$$
\partial_{t} \mathbf{w}+\partial_{t} u \mathbf{p}^{s s}=\frac{1}{\epsilon} A\left(\mathbf{w}+u \mathbf{p}^{s s}\right)+\mathcal{L}\left(\mathbf{w}+u \mathbf{p}^{s s}\right) .
$$

Using equation (3.8) and the fact that $\mathbf{p}^{s s}$ is in the right null space of $A$, we obtain

$$
\partial_{t} \mathbf{w}=\frac{1}{\epsilon} A \mathbf{w}+\left(\mathbb{I}_{3}-\mathbf{p}^{s s} \psi^{T}\right) \mathcal{L}\left(\mathbf{w}+u \mathbf{p}^{s s}\right),
$$

where $\mathbb{I}_{3}$ is the $3 \times 3$ identity matrix. Now introduce an asymptotic expansion for $\mathbf{w}$ of the form ${ }^{1}$

$$
\mathbf{w} \sim \mathbf{w}_{0}+\epsilon \mathbf{w}_{1}+\epsilon^{2} \mathbf{w}_{2}+\ldots
$$

After substituting this expansion into (3.10) and collecting $\mathcal{O}\left(\epsilon^{-1}\right)$ terms we obtain the equation for $\mathbf{w}_{0}$

$$
A \mathbf{w}_{0}=0 .
$$

Since $\mathbf{w}$ is in the orthogonal complement of the left nullspace of $A$, it follows that $\mathbf{w}_{0}=0$. Now collecting terms of $\mathcal{O}(1)$ yields the equation

$$
A \mathbf{w}_{1}=-\left(\mathbb{I}_{3}-\mathbf{p}^{s s} \psi^{T}\right) \mathcal{L}\left(u \mathbf{p}^{s s}\right) .
$$

Although the matrix $A$ is singular, the orthogonal projection operator $\left(\mathbb{I}_{3}-\right.$ $\mathbf{p}^{s s} \psi^{T}$ ) ensures that the right-hand side of the above equation is in the range of $A$. By the Fredholm alternative theorem a solution $\mathbf{w}_{1}$ exists. Writing out the equations in full, we have

$$
\begin{aligned}
\frac{1}{\gamma b_{+}}\left(1-\frac{1}{\gamma b_{+}}\right) \partial_{x} u+\frac{1}{\gamma^{2} b_{+} b_{-}} \partial_{x} u-\frac{k(x)}{\gamma^{2} a b_{+}} u & =-b_{+} w_{1,+}+a w_{1,0} \\
-\frac{1}{\gamma b_{-}}\left(1-\frac{1}{\gamma b_{-}}\right) \partial_{x} u-\frac{1}{\gamma^{2} b_{+} b_{-}} \partial_{x} u-\frac{k(x)}{\gamma^{2} a b_{-}} u & =-b_{-} w_{1,-}+a w_{1,0} \\
-\frac{1}{\gamma^{2} a b_{+}} \partial_{x} u+\frac{1}{\gamma^{2} a b_{-}} \partial_{x} u+\left(1-\frac{1}{\gamma a}\right) \frac{k(x)}{\gamma a} u & =b_{+} w_{1,+}+b_{-} w_{1,-}-2 a w_{1,0} .
\end{aligned}
$$

\footnotetext{
${ }^{1}$ For simplicity, we choose $\sigma_{m}=p_{m}^{\text {ss }}$ in equation (2.1) so that the initial state lies in the slow manifold. This allows us to neglect terms that converge exponentially fast on to the slow manifold. Such terms could be taken into account by introducing an additional fast time-scale $\tau=t / \varepsilon$.
} 
This rank 2 system can be solved up to the arbitrary element $w_{0,1}$ using Gaussian elimination. We have

$$
\mathbf{w} \sim \epsilon\left(\begin{array}{c}
\frac{k(x)}{a b_{+}^{2} \gamma^{2}} u-\frac{1-V_{0}}{b_{+}^{2} \gamma} \partial_{x} u+\frac{a \Omega}{b_{+}} \\
\frac{k(x)}{a b_{-}^{2} \gamma^{2}} u+\frac{1+V_{0}}{b_{-}^{2} \gamma} \partial_{x} u+\frac{a \Omega}{b_{-}}
\end{array}\right)
$$

where we have set $\Omega=w_{0,1}$ and

$$
V_{0}=\frac{1}{\gamma}\left(\frac{1}{b_{+}}-\frac{1}{b_{-}}\right) .
$$

We can determine $\Omega$ by imposing the condition $\psi^{T} \mathbf{w}=0$. This gives

$$
a \Omega=-\frac{k(x)}{a \gamma^{3}}\left(\frac{1}{b_{+}^{2}}+\frac{1}{b_{-}^{2}}\right) u+\frac{1}{\gamma^{2}}\left(\frac{1-V_{0}}{b_{+}^{2}}-\frac{1+V_{0}}{b_{-}^{2}}\right) \partial_{x} u
$$

Substituting equations (3.15) and (3.16) into (3.8) yields the Fokker-Plank equation

$$
\frac{\partial u}{\partial t}=-\Lambda u-V \frac{\partial u}{\partial x}+D \frac{\partial^{2} u}{\partial x^{2}}
$$

where $\Lambda=\lambda \chi(x-X)$ and

$$
\begin{aligned}
\lambda & =\frac{k_{0}}{a \gamma}+\epsilon \frac{k_{0}^{2}}{a^{2} \gamma^{3}}\left(\frac{1}{b_{+}^{2}}+\frac{1}{b_{-}^{2}}\right) \\
V & =V_{0}+\epsilon \frac{k_{0} \chi(x-X)}{a \gamma^{2}}\left(\frac{1}{b_{+}^{2}}-\frac{1}{b_{-}^{2}}+\frac{1-2 V_{0}}{b_{+}^{2}}-\frac{1+2 V_{0}}{b_{-}^{2}}\right) \\
D & =\epsilon\left(\frac{\left(1-V_{0}\right)^{2}}{\gamma b_{+}^{2}}+\frac{\left(1+V_{0}\right)^{2}}{\gamma b_{-}^{2}}\right) .
\end{aligned}
$$

The leading order behavior is obtained by taking $\lambda=\lambda_{0}=k_{0} / a \gamma$ and $V=V_{0}$. The probability density function $u$ is the total probability of being in any motor state at position $x$ and time $t$, given that the particle was initially injected on to the track at $x=0$. The initial condition is thus $u(x, 0)=\delta(x)$. Equation (3.17) is supplemented by a reflecting boundary condition at $x=0$ and an absorbing boundary condition at $x=L$ :

$$
-V u(0)+\left.D \frac{\partial u}{\partial x}\right|_{x=0}=0, \quad u(L)=0 .
$$

These boundary conditions follow from substituting $\mathbf{p}=u \mathbf{p}^{\mathrm{ss}}+\varepsilon \mathbf{w}_{1}$ into the boundary conditions (2.4) of the corresponding 3 -state master equation and using equations (3.15) and (3.16). Note that the Fokker-Planck equation has to be solved separately in the three domains $0 \leq x \leq X-1, X-1 \leq x \leq$ $X+1$ and $X+1 \leq x \leq L$. The solution involves six unknown constants, and hence, we require six supplementary conditions. Two are obtained from 
the boundary conditions and the other four from imposing continuity of $u$ and $\partial_{x} u$ at $x=X \pm 1$. At first sight, these continuity conditions appear to be inconsistent with the observation that $p_{0}(x, t)$ is discontinuous at $x=X \pm 1$. However, the discontinuities are $\mathcal{O}(\varepsilon)$ and are thus incorporated into the higher-order corrections $\mathbf{w}_{j}$. The $\varepsilon$ dependence of the continuous solution $u$ reflects the separation of time scales in a multi-scale analysis.

\subsection{Generalized reduction}

A general system of $n$ reaction-hyperbolic master equations may be reduced in a similar manner, provided that the transition rates between the internal motor states are fast compared to the speeds of the motor complex. Consider the following (non-dimensionalized) master equation governing the time evolution of the probability density function $\mathbf{p}(x) \in \mathbb{R}^{n}$ with $x \geq 0$ :

$$
\partial_{t} \mathbf{p}=\frac{1}{\epsilon} A \mathbf{p}+\mathcal{L}(\mathbf{p})
$$

where $A \in \mathbb{R}^{n \times n}$ is the matrix containing the transition rates between each of the $n$ internal motor states and the differential operator $\mathcal{L}$ has the form

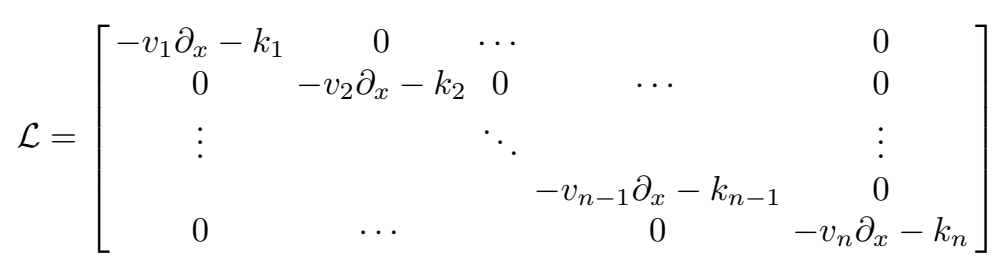

We assume that the matrix $A$ is irreducible and is conservative so that $\psi=(1,1, \cdots, 1)^{T}$ is in the nullspace of $A^{T}$. We also assume that $A$ has one zero eigenvalue and that the remaining eigenvalues are real and negative. Let $\mathbf{p}^{s s} \in N(A)$ and choose $\mathbf{p}^{s s}$ so that $\psi^{T} \mathbf{p}^{s s}=1$. We take $v_{i}>0$ for $i=1, \ldots, m$ and $v_{i} \leq 0$ for $i=m+1, \ldots, n$ with $m>0$. The requirement that the transport is biased in the anterograde direction is

$$
V_{0} \equiv \sum_{j=1}^{n} v_{j} p_{j}^{s s}>0
$$

The master equation (3.22) is supplemented by absorbing boundary conditions at $x=L$

$$
p_{i}(L, t)=0 \text { for } i=m+1, \ldots n,
$$

a reflecting boundary condition at $x=0$

$$
\sum_{i=1}^{n} v_{i} p_{i}(0, t)=0,
$$


and the auxiliary boundary conditions

$$
\frac{p_{i}(0, t)}{p_{1}(0, t)}=\frac{p_{i}^{\mathrm{ss}}}{p_{1}^{\mathrm{ss}}}, \quad i=2, \ldots, m
$$

The initial conditions are taken to be $p_{i}(x, 0)=p_{i}^{\mathrm{ss}} \delta(x)$.

Proceeding along identical lines to the 3 -state model, we introduce the decomposition $\mathbf{p}=u \mathbf{p}^{s s}+\mathbf{w}$ where $u \equiv \psi^{T} \mathbf{p}$ and $\psi^{T} \mathbf{w}=0$. Multiplying both sides of (3.22) by $\psi^{T}$ yields the equation

$$
\partial_{t} u=\psi^{T} \mathcal{L}\left(u \mathbf{p}^{s s}+\mathbf{w}\right) .
$$

Substituting $\mathbf{p}=u \mathbf{p}^{s s}+\mathbf{w}$ into (3.22) and performing an asymptotic expansion of $\mathbf{w}$ we find that $\mathbf{w} \sim \epsilon \mathbf{w}_{1}$ with

$$
A \mathbf{w}_{1}=-\left(\mathbb{I}_{n}-\mathbf{p}^{s s} \psi^{T}\right) \mathcal{L}\left(u \mathbf{p}^{s s}\right),
$$

where $\mathbb{I}_{n}$ is the $n \times n$ identity matrix. The orthogonal projection $\mathbb{I}_{n}-\mathbf{p}^{s s} \psi^{T}$ ensures that the right-hand side of the above equation is in the range of $A$, and we obtain a unique solution by requiring that $\psi^{T} \mathbf{w}_{1}=0$.

Substituting $\mathbf{w} \sim \epsilon \mathbf{w}_{1}$ into (3.28) yields

$$
\partial_{t} u=-\sum_{j=1}^{n} k_{j}\left(p_{j}^{s s}+\epsilon w_{1, j}\right) u-\sum_{j=1}^{n}\left(v_{j} p_{j}^{s s}\right) \partial_{x} u-\epsilon \sum_{j=1}^{n} v_{j} \partial_{x} w_{1, j}
$$

The components of $\mathbf{w}_{1}$ are linear combinations of $u$ and $\partial_{x} u$ so that we can write them as

$$
w_{j}=\Omega_{j} u-\theta_{j} \partial_{x} u
$$

where $\Omega_{j}$ and $\theta_{j}, j=1, \ldots, n$, are $u$-independent. Collecting $\partial_{x} u$ terms in (3.29) yields an equation for $\theta=\left(\theta_{1}, \ldots, \theta_{n}\right)^{T}$,

$$
A \theta=-\left(\left(V_{0}-v_{1}\right) p_{1}^{s s}, \ldots,\left(V_{0}-v_{n}\right) p_{n}^{s s}\right)^{T} .
$$

The condition $\psi^{T} \mathbf{w}_{1}=0$ implies that $\psi^{T} \theta=0$ and hence we can solve for $\theta$ uniquely. Thus, to leading order, we obtain the Fokker-Plank equation (3.17) with

$$
\begin{aligned}
& \Lambda=\sum_{j=1}^{n} k_{j} p_{j}^{s s}+\mathcal{O}(\epsilon) \\
& V=\sum_{j=1}^{n} v_{j} p_{j}^{s s}+\mathcal{O}(\epsilon) \\
& D=\epsilon \sum_{j=1}^{n} v_{j} \theta_{j}+\mathcal{O}\left(\epsilon^{2}\right) .
\end{aligned}
$$

It can be checked that the associated boundary conditions (3.21) are consistent with equations (3.25)-(3.27) to leading order in $\varepsilon$. 


\section{Hitting probability $\Pi$ and conditional MFPT $T$}

In this section we use the Fokker-Planck equation (3.17) to calculate the hitting probability $\Pi$ and MFPT $T$ for finding the target, and then compare our analytical results with numerical results obtained from Monte Carlo simulations of the full 3-state model given by the master equation (2.2).

\subsection{Generating function and Laplace transforms}

Let $\mathbb{P}(t)=\int_{0}^{L} u(x, t) d x$ be the total probability that the particle is still located in the domain $0<x<L$ at time $t$. Integrating equation (3.17) with respect to $x$ and using the boundary conditions (3.21), we have

$$
\frac{\partial \mathbb{P}}{\partial t}=-\lambda \int_{X-1}^{X+1} u(x, t) d x+\left.D \frac{\partial u}{\partial x}\right|_{x=L} .
$$

It follows that the total flux into the target is

$$
J(t)=\lambda \int_{X-1}^{X+1} u(x, t) d x,
$$

The hitting probability that the particle succeeds in finding the target having started at $x=0$ at time $t=0$ is then

$$
\Pi=\int_{0}^{\infty} J(t) d t
$$

and the corresponding conditional MFPT is

$$
T=\frac{\int_{0}^{\infty} t J(t) d t}{\int_{0}^{\infty} J(t) d t} .
$$

There are two alternative methods for calculating $\Pi$ and $T$, one based on Laplace transforms and the other based on solving the corresponding backwards Fokker-Planck equation [35]. We will follow the former approach here. First, consider the Laplace transform of the probability flux $J$,

$$
\widetilde{J}(s)=\int_{0}^{\infty} \mathrm{e}^{-s t} J(t) d t
$$

Taylor expanding the integral with respect to the Laplace variable $s$ shows that

$$
\begin{aligned}
\widetilde{J}(s) & =\int_{0}^{\infty} J(t)\left[1-s t+s^{2} t^{2} / 2-\ldots\right] \\
& =\Pi\left[1-s T+\frac{s^{2}}{2} T^{(2)}-\ldots\right],
\end{aligned}
$$


assuming that the moments

$$
T^{(n)}=\frac{\int_{0}^{\infty} t^{n} J(t) d t}{\int_{0}^{\infty} J(t) d t}
$$

are finite. Thus, $\widetilde{J}(s)$ can be viewed as a generating function for the moments of the conditional first passage time distribution [35]. Equations (4.2) and (4.5) imply that

$$
\widetilde{J}(s)=\lambda \int_{X-1}^{X+1} \widetilde{U}(x, s) d x,
$$

where $\widetilde{U}(x, s)$ is the Laplace transform of $u(x, t)$. Hence, we can proceed by solving the Laplace transformed Fokker-Planck equation to determine $\widetilde{U}(x, s)$. Substituting the result into equation (4.8) and Taylor expanding with respect to $s$ then allows us to extract $\Pi$ and $T$ using equation (4.6).

Laplace transforming equation (3.17) under the initial condition $u(x, 0)=$ $\delta(x)$ gives

$$
D \partial_{x}^{2} \widetilde{U}(x, s)-V \partial_{x} \widetilde{U}(x, s)-(\lambda \chi(x-X)+s) \widetilde{U}(x, s)=-\delta(x) .
$$

The delta function at $x=0$ can be incorporated into the corresponding boundary condition

$$
\left.D \partial_{x} \widetilde{U}(x, s)\right|_{x=0}-V \widetilde{U}(0, s)=-1 .
$$

Equation (4.9) is solved separately in the three regions $0 \leq x \leq X-1$, $X-1 \leq x \leq X+1$ and $X+1 \leq x \leq L$. Each solution is then matched by requiring continuous differentiability at $x=X \pm 1$. In region I $(0 \leq x \leq$ $X-1)$ we have $\chi(x-X)=0$ so that the solution has the form

$$
\widetilde{U}_{I}(x, s)=A_{I} \mathrm{e}^{\mu_{+}(s) x}+B_{I} \mathrm{e}^{\mu_{-}(s) x},
$$

where

$$
\mu_{ \pm}(s)=\frac{1}{2}\left(\xi \pm \sqrt{\xi^{2}+4 s / D}\right), \quad \xi=\frac{V}{D} .
$$

Imposing the inhomogeneous boundary condition at $x=0$ implies that

$$
B_{I}=-A_{I} \frac{\mu_{+}(s)-\xi}{\mu_{-}(s)-\xi}-\frac{1}{D\left(\mu_{-}(s)-\xi\right)} .
$$

Similarly, in region III $(X+1 \leq x \leq L)$ we have a solution of the form

$$
\widetilde{U}_{I I I}(x, s)=A_{I I I} \mathrm{e}^{\mu_{+}(s) x}+B_{I I I} \mathrm{e}^{\mu_{-}(s) x} .
$$

Imposing the right boundary condition $\widetilde{U}_{I I I}(L, s)=0$ requires that

$$
B_{I I I}=-A_{I I I} \mathrm{e}^{\left(\mu_{+}(s)-\mu_{-}(s)\right) L} .
$$


In region II $(X-1 \leq x \leq X+1)$ for which $\chi(x-X)=1$, we have solutions of the form

$$
\widetilde{U}_{I I}(x, s)=A_{I I} \mathrm{e}^{\nu_{+}(s) x}+B_{I I} \mathrm{e}^{\nu_{-}(s) x},
$$

where

$$
\nu_{ \pm}(s)=\frac{1}{2}\left(\xi \pm \sqrt{\xi^{2}+4(\lambda+s) / D}\right)
$$

The remaining constants are determined by imposing continuity of $\widetilde{U}$ and $\partial_{x} \widetilde{U}$ at $x=X \pm 1$. After solving the resulting four equations we obtain the unknown constants necessary to determine the solution in the target region. It is convenient to introduce the functions

$$
\begin{aligned}
& \Psi(X, s)=\mathrm{e}^{\mu_{+}(s)(X-1)}-\left(\frac{\mu_{+}(s)-\xi}{\mu_{-}(s)-\xi}\right) \mathrm{e}^{\mu_{-}(s)(X-1)} \\
& \Phi(X, s)=\mathrm{e}^{\mu_{+}(s)(X+1-L)}-\mathrm{e}^{\mu_{-}(s)(X+1-L)} \\
& F(X, s)=-\frac{\mathrm{e}^{\mu_{-}(s) X}}{\left(\mu_{-}(s)-\xi\right) D} .
\end{aligned}
$$

Then

$$
\begin{aligned}
& A_{I I}=\frac{1}{\Omega}\left[\left(\partial_{X} \Psi\right) F-\Psi\left(\partial_{X} F\right)\right] D_{-} \\
& B_{I I}=\frac{-1}{\Omega}\left[\left(\partial_{X} \Psi\right) F-\Psi\left(\partial_{X} F\right)\right] D_{+},
\end{aligned}
$$

where

$$
\begin{gathered}
\Omega(X, s)=\Psi(X, s)\left[\nu_{-}(s) D_{+}(X, s) \mathrm{e}^{\nu_{-}(s)(X-1)}-\nu_{+}(s) D_{-}(X, s) \mathrm{e}^{\nu_{+}(s)(X-1)}\right] \\
-\partial_{X} \Psi(X, s)\left[D_{+}(X, s) \mathrm{e}^{\nu_{-}(s)(X-1)}-D_{-}(X, s) \mathrm{e}^{\nu_{+}(s)(X-1)}\right],
\end{gathered},
$$

and

$$
D_{ \pm}(X, s)=\mathrm{e}^{\nu_{ \pm}(s)(X+1)}\left[\Phi(X, s) \nu_{ \pm}(s)-\partial_{X} \Phi(X, s)\right] .
$$

The hitting probability $\Pi$ and MFPT can now be calculated by substituting $\widetilde{U}=\widetilde{U}_{I I}$ into equation (4.8) for the generating function $\widetilde{J}(s)$, and setting

$$
\Pi=\widetilde{J}(0), \quad T=-\frac{\widetilde{J}^{\prime}(0)}{\widetilde{J}(0)} .
$$



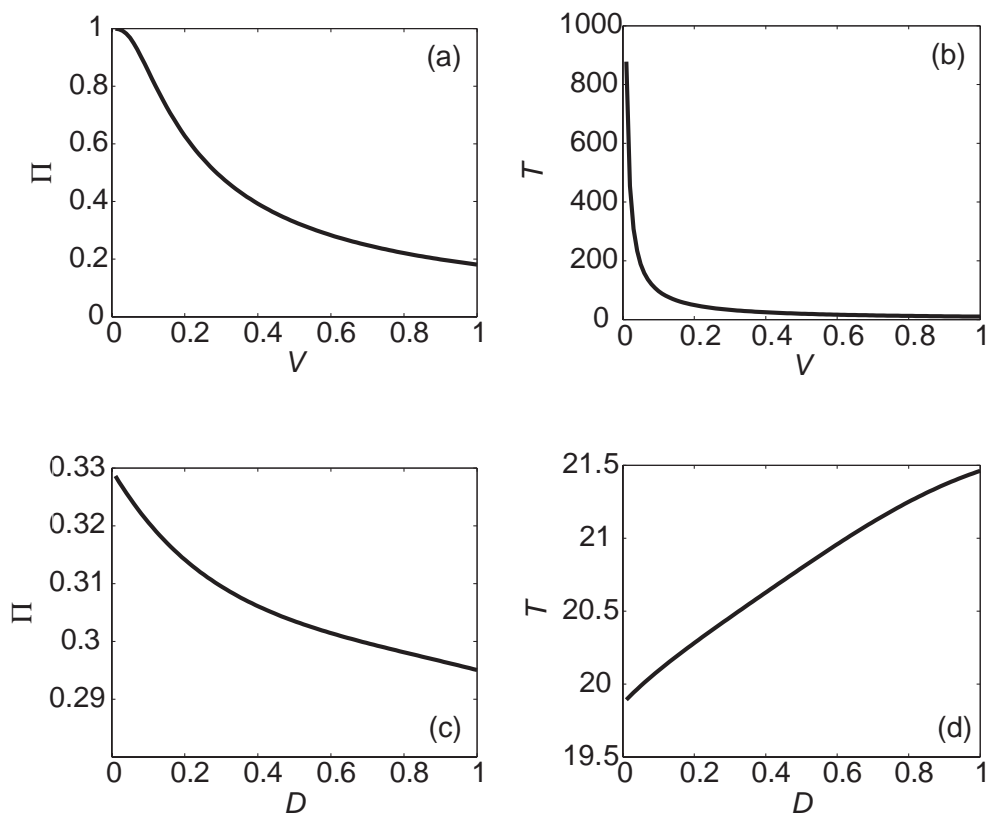

Fig. 3 (a,b) Plots of hitting probability $\Pi$ and MFPT $T$ as a function of the drift $V$ for fixed diffusivity $D=0.005$. (c,d) Corresponding plots as a function of $D$ for fixed $V=0.5$. Other parameter values are $X=10, L=20, \lambda=0.1$. All parameters are non-dimensionalized by taking the length-scale to be the typical size $l$ of a target and the time-scale to be $l / v$ where $v$ is the typical speed of a molecular motor.
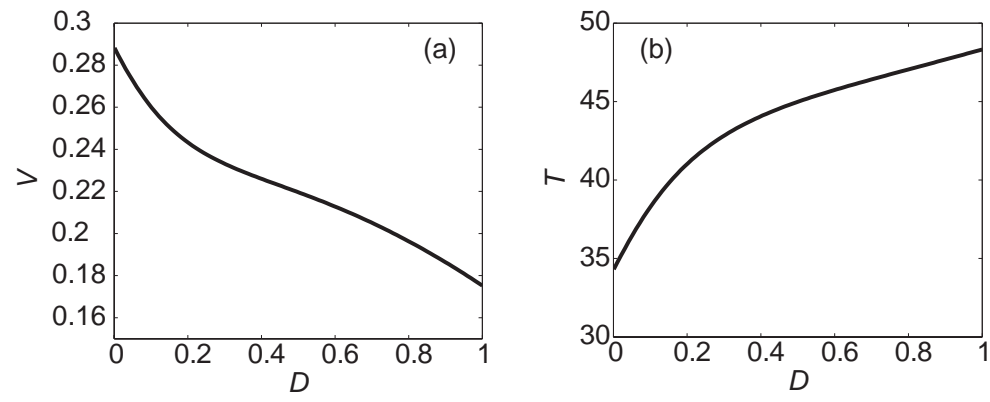

Fig. 4 (a) Plot of drift $V$ as a function of $D$ for $\lambda=0.1$ and fixed hitting probability $\Pi_{0}=0.5$. (b) Corresponding plot of the MFPT $T$.

\subsection{Dependence on effective diffusivity $D$ and drift $V$}

Having calculated the hitting probability $\Pi$ and MFPT $T$ for the reduced Fokker-Planck equation (3.17), we can use these expressions to explore how $\Pi$ and $T$ depend on the effective diffusivity $D$ and drift $V$ (assuming that $\lambda$ is fixed). The results of our analysis are shown in Fig 3. It can be seen 
that both $\Pi$ and $T$ are monotonically decreasing functions of $V$ for fixed $D$. On the other hand, $\Pi$ is a decreasing function of $D$, whereas $T$ is an increasing function of $D$ for fixed $V$. This suggests that it is advantageous to reduce the diffusivity as much as possible, since this generates a random search that has a high hitting probability and a relatively small MFPT. However, it should be noted that $D$ and $V$ have a complicated dependence on the specific parameters of the underlying biophysical motor transport model, so that changes in $D$ and $V$ are generally going to be correlated. Another way to characterize the efficiency of the random search is to fix the hitting probability to some value $\Pi=\Pi_{0}$ and to solve the equation $\Pi_{0}=\Pi(\lambda, V, D)$ for $V[9]$. We can then determine $T\left(\lambda, V\left(D ; \Pi_{0}, \lambda\right), D\right)$ as a function of $D$ with $\lambda$ and $\Pi_{0}$ fixed. The results are shown in Fig 4, which illustrate how the MFPT $T$ can be reduced by reducing the diffusivity for a fixed hitting probability.

\subsection{Comparison with 3-state model}
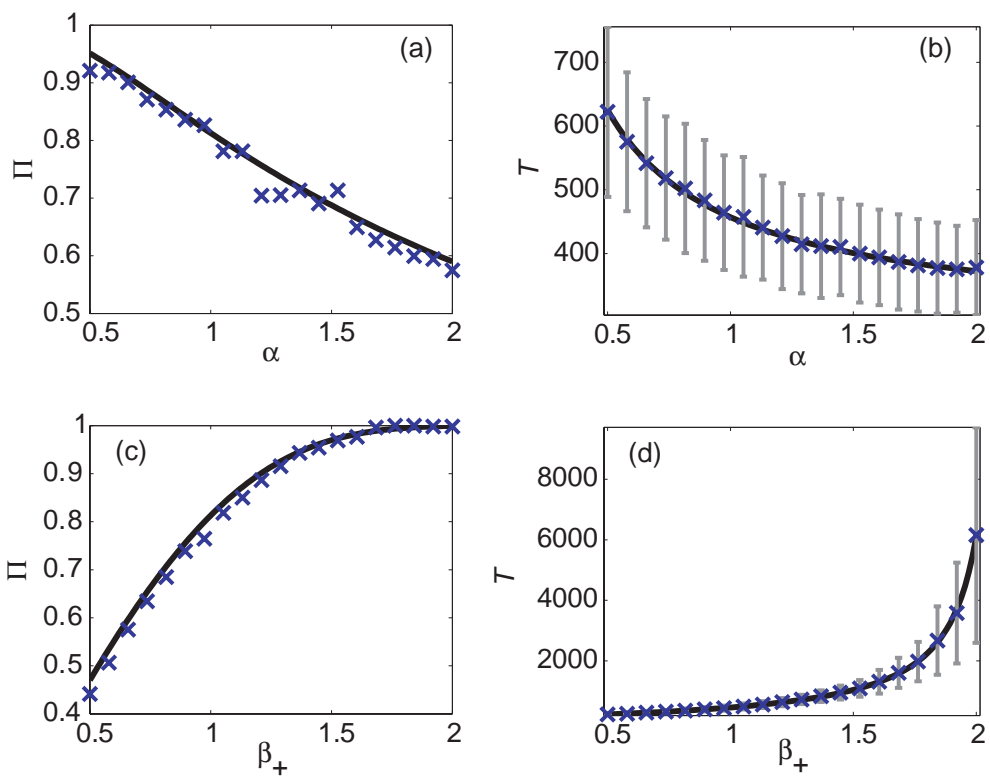

Fig. 5 Comparison of analytical results based on the Fokker-Planck equation (solid curves) with Monte Carlo simulations of the full 3-state model given by the master equation (2.2) (discrete points $\mathrm{x}$ ). (a,b) Plots of the hitting probability $\Pi$ and the MFPT $T$ as functions of the transition rate $\alpha$ for fixed $\beta_{+}=1 \mathrm{~s}^{-1}$. Other parameter values are $\beta_{-}=1 \mathrm{~s}^{-1}, \kappa=0.05 \mathrm{~s}^{-1}, v=0.1 \mu \mathrm{ms}^{-1}, X=10 \mu \mathrm{m}$, $L=20 \mu \mathrm{m}, l=1 \mu \mathrm{m}$. Error bars denote the standard deviation of the conditional first passage time obtained in the Monte Carlo simulations. (c,d) Corresponding plots as a function of $\beta_{+}$for fixed $\alpha=1 s^{-1}$. 
If the quasi-steady state reduction is used to express $D, V$ and $\lambda$ in terms of the transition rates and target absorption rates of the underlying motor transport master equation, then the dependence of $\Pi$ and $T$ on the modelspecific parameters can be determined. We will illustrate this for the 3 -state model introduced in section 2 , and then consider a more complex multiple motor model in section 5. In the former case, we use equations (3.18)(3.20) to express the generic parameters $\lambda, V, D$ in terms of the motor state transition rates $\alpha, \beta_{ \pm}$, the target absorption rate $\kappa$ and the motor speed $v$. We take the small parameter $\epsilon=v /(\alpha l)$ so that $a=1$ and $b_{ \pm}=\beta_{ \pm} / \alpha$. For concreteness, we follow our previous work [9] and take parameter values extracted from experimental studies of mRNA transport in dendrites [25, $37,13]$. Thus we consider the following baseline values for the transition rates and motor speed: $\alpha=1 s^{-1}, \beta_{+}=1 s^{-1}, \beta_{-}=2 s^{-1}, \kappa=0.05 s^{-1}$ and $v=0.1 \mu \mathrm{ms}^{-1}$. It follows that for a target of size $l=1 \mu \mathrm{m}$, we have $\epsilon=0.1$ and $a=b_{+}=1, b_{-}=2$. In Fig. 5 we compare our analytical results for $\Pi$ and $T$ based on the quasi-steady state reduction of the 3 -state model, with Monte Carlo simulations of the full model. It can be seen that there is excellent agreement between the two.

\section{Multiple molecular motors}

The simplified model of bidirectional transport presented in section 2 assumes that there is a single motor complex that can be in several different velocity states. In this section we consider a more realistic model in which the directed motion of a cargo is a result of the combined action of multiple motors attached to the cargo; the current velocity state is determined by the subset of motors currently bound to a microtubule track [44]. As we highlighted in the introduction, microtubules are polarized filaments with biophysically distinct $(+)$ and $(-)$ ends, and this polarity determines the preferred direction in which an individual molecular motor moves. For example, kinesin moves towards the $(+)$ end whereas dynein moves towards the $(-)$ end. In axons and dendrites that are located far from the cell body one finds that microtubule filaments all have the same polarity, with the $(+)$ end oriented away from the cell body. This suggests a model of bidirectional transport in which kinesin and dynein motors transport a cargo in opposite directions along a single track. On the other hand, dendritic microtubules located close to the cell body tend to have mixed polarities [1], suggesting a model in which motors of the same directional preference are distributed among two parallel microtubules of opposite polarity.

In both of the above scenarios, there has to be some mechanism for coordinating the action of the various motors as part of a larger motor complex. One possibility is that the motors interact through a tug-of-war competition, where individual motors influence each other through the force they exert on the cargo $[44,31]$. When a force is exerted on a motor opposite to its preferred direction, it is more likely to detach from its microtubule. Ultimately the motion of the cargo is determined by the random attachments 
and force-dependent detachments from the microtubule of each motor in the motor complex. A corresponding biophysical model of motor competition has been developed, in which the transitions between the different internal motor states are described in terms of a discrete Markov process [31]. Here we extend this tug-of-war model in order to account explicitly for the position of the motor complex along a one-dimensional track. The resulting master equation then becomes a system of linear reaction hyperbolic equations. For concreteness, we consider the case of multiple kinesin motors engaged in a tug-of-war competition by pulling a cargo along a track consisting of two parallel microtubules with opposite polarity, see Fig. 6 . Internal states of the motor complex are determined by the number of motors bound to each microtubule. Each internal state represents a different net force placed on the cargo and therefore a different cargo velocity. In order to formulate the model within the context of directed intermittent search, we define the search phase as any motor state with zero net cargo velocity. We then apply the general quasi-steady state reduction of section 3.2 in order to obtain the parameters $\lambda, V$, and $D$ and use this to calculate the hitting probability and MFPT as defined in section 4.

\subsection{Tug-of-war model of intermittent search}

Suppose that a certain cargo is transported via $N_{q}$ anterograde kinesin motors and $N_{r}$ retrograde kinesin motors via a pair of microtubule filaments of opposite polarity, see Fig 6. (A motor is labeled according to its direction of motion in the absence of an applied force). At a given time $t$, the internal state of the cargo-motor complex is fully characterized by the numbers $n_{q}$ and $n_{r}$ of anterograde and retrograde motors that are bound to a microtubule and thus actively pulling on the cargo. The binding and unbinding rates and the cargo velocities are obtained from the tug-of-war model [31]. This assumes that the motors act independently other than exerting a load on motors bound to the opposing microtubule, with identical motors sharing the load. Thus the properties of the motor complex can be determined from the corresponding properties of the individual motors together with a specification of the effective load on each motor. Let us begin by considering a single kinesin motor. When bound to a microtubule, each motor has a load-dependent velocity (taken to be positive when directed towards the $(+)$ end)

$$
v(F)= \begin{cases}v_{f}\left(1-F / F_{s}\right) & \text { for } F \leq F_{s} \\ v_{b}\left(1-F / F_{s}\right) & \text { for } F \geq F_{s}\end{cases}
$$

where $F$ is the applied force, $F_{s}$ is the stall force satisfying $v\left(F_{s}\right)=0, v_{f}$ is the $(+)$ end motor velocity in the absence of an applied force, and $v_{b}$ is the $(-)$ end motor velocity when the applied force exceeds the stall force. The unbinding rate of an individual motor from its microtubule is assumed to increase exponentially with the applied force $F$

$$
\varepsilon(F)=\varepsilon_{0} \mathrm{e}^{F / F_{d}},
$$




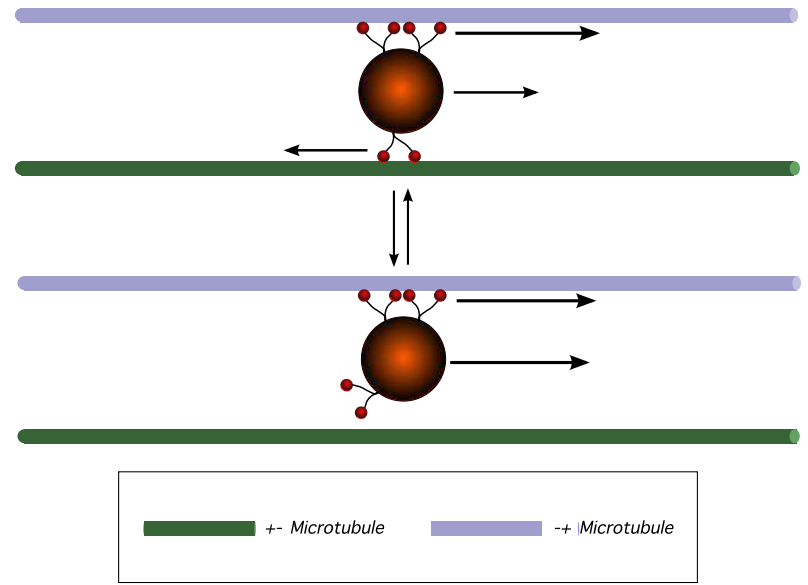

Fig. 6 The tug-of-war model of bidirectional motor transport for a cargo with two anterograde motors and a single retrograde motor moving along a track consisting of a pair of microtubules with opposite polarity. Two out of the six possible motor states are shown. Individual motors may attach and detach from the microtubule changing the net velocity of the cargo.

where $F_{d}$ is the detachment force. On the other hand, the binding rate is taken to be independent of load:

$$
\zeta(F)=\zeta_{0}
$$

Let $F_{c}$ denote the net load on the set of anterograde motors, which is taken to be positive when pointing in the retrograde direction. It follows that a single anterograde motor feels the force $F_{c} / n_{q}$, whereas a single retrograde motor feels the opposing force $-F_{c} / n_{r}$. Equations (5.2) and (5.3) imply that the binding and unbinding rates for the anterograde and retrograde motors are given by

$$
\begin{aligned}
\varepsilon_{l}\left(n_{l}, F_{c}\right) & =n_{l} \varepsilon_{0} \exp \left[\frac{F_{c}}{n_{l} F_{d}}\right] \\
\zeta_{l}\left(n_{l}\right) & =\left(N_{l}-n_{l}\right) \zeta_{0} .
\end{aligned}
$$

for $l=q, r$. The cargo force $F_{c}$ is determined by the condition that all the motors move with the same cargo velocity $v_{c}$. Suppose that $n_{q}>n_{r}$ so that the net motion is in the anterograde direction, which is taken to be positive. Equation (5.1) implies that

$$
v_{c}=v_{f}\left(1-F_{c} /\left(n_{q} F_{s}\right)\right)=-v_{b}\left(1-F_{c} /\left(n_{r} F_{s}\right)\right) .
$$

The corresponding expression when $n_{q}<n_{r}$ is found by interchanging $v_{f}$ and $v_{b}$. We thus obtain a unique solution for the load $F_{c}$ and cargo velocity $v_{c}[31]:$

$$
F_{c}=F_{c}\left(n_{q}, n_{r}\right)=\left(\mathcal{F} n_{q}+(1-\mathcal{F}) n_{r}\right) F_{s}
$$


where

$$
\mathcal{F}= \begin{cases}\frac{n_{r} v_{f}}{n_{r} v_{f}+n_{q} v_{b}} & \text { for } n_{q}>n_{r} \\ \frac{n_{r} v_{b}}{n_{r} v_{b}+n_{q} v_{f}} & \text { for } n_{q}<n_{r}\end{cases}
$$

The corresponding cargo velocity is

$$
v_{c}=v_{c}\left(n_{q}, n_{r}\right)= \begin{cases}\frac{n_{q}-n_{r}}{n_{q} / v_{f}+n_{r} / v_{b}} & \text { for } n_{q}>n_{r} \\ \frac{n_{q}-n_{r}}{n_{q} / v_{b}+n_{r} / v_{f}} & \text { for } n_{q}<n_{r} .\end{cases}
$$

The original tug-of-war model [31] considers the stochastic dynamics of the internal state of the motor complex, without specifying the spatial position of the cargo along the track. In order to apply the model to the problem of directed intermittent search, it is necessary to construct a master equation for the probability density $p\left(n_{q}, n_{r}, x, t\right)$ that the cargo is in the internal state $\left(n_{q}, n_{r}\right)$ and has position $x$ at time $t$. Such a master equation can be written in the general reaction-hyperbolic form (3.22) by introducing the label $i\left(n_{q}, n_{r}\right)=\left(N_{q}+1\right) n_{r}+\left(n_{q}+1\right)$ and setting $p\left(n_{q}, n_{r}, x, t\right)=$ $p_{i\left(n_{q}, n_{r}\right)}(x, t)$. We then have an $n$-component probability vector $\mathbf{p} \in \mathbb{R}^{n}$ with $n=\left(N_{q}+1\right)\left(N_{r}+1\right)$. The components $a_{i j}, i, j=1, \ldots, n$, of the state transition matrix $A$ are given by the corresponding binding/unbinding rates of equations (5.4) and (5.5). That is, setting $i=i\left(n_{q}, n_{r}\right)$, the non-zero offdiagonal terms are

$$
\begin{gathered}
a_{i j}=\zeta_{q}\left(n_{q}-1\right) \text { for } j=i\left(n_{q}-1, n_{r}\right) \\
a_{i j}=\zeta_{r}\left(n_{r}-1\right), \text { for } j=i\left(n_{q}, n_{r}-1\right) \\
a_{i j}=\varepsilon_{q}\left(n_{q}+1, F_{c}\right), \text { for } j=i\left(n_{q}+1, n_{r}\right)
\end{gathered}
$$

and

$$
a_{i j}=\varepsilon_{r}\left(n_{r}+1, F_{c}\right), \text { for } j=i\left(n_{q}, n_{r}+1\right) .
$$

The diagonal terms are then given by $a_{i i}=-\sum_{j \neq i} a_{i j}$. The linear operator $\mathcal{L}$ is defined according to (3.23) with the state-dependent velocities

$$
v_{i}=v_{c}\left(n_{q}, n_{r}\right), \quad i=i\left(n_{q}, n_{r}\right)
$$

and the target detection rates

$$
k_{i}(x)= \begin{cases}k_{0} \chi(x-X) & \text { if } v_{i}=0 \\ 0 & \text { otherwise. }\end{cases}
$$

Finally, we non-dimensionalize the master equation by fixing units such that one of the velocities and one of the transition rates are unity. For concreteness, we take $\zeta_{r}(0)=1$ and $v_{c}\left(N_{q}, N_{r}\right)=1$ so that the small parameter in equation $(3.22)$ is

$$
\epsilon=\frac{v_{c}\left(N_{q}, N_{r}\right)}{\zeta_{r}(0) l}
$$

where $l$ is the size of the target. 


\subsection{Quasi-steady state analysis of model}

Having formulated the tug-of-war model of directed intermittent search in terms of the generalized master equation (3.22), we can now carry out the quasi-steady state reduction outlined in section 3.2. We first numerically calculate the steady state probability distribution $\mathbf{p}^{s s}$ using a singular valued decomposition (SVD) of the matrix $A$ to obtain a vector spanning the nullspace of $A$, normalized so that $\sum_{j} p_{j}^{s s}=1$. We can then use equations (3.33) and (3.34) to compute the detection rate $\lambda$ and the drift velocity $V$. However, in order to calculate the effective diffusivity $D$ using (3.35), we have to compute the vector $\theta$ by solving equation (3.32), which has the general form $A \theta=b$. The standard numerical method for solving a rank deficient linear system using SVD must be modified slightly. The FredholmAlternative theorem tells us that a solution to equation (3.32) exists but is not unique. In the case of a standard least squares solution, uniqueness is obtained by requiring the solution to be orthogonal to the nullspace of $A$. However, in our case a unique solution must be obtained by requiring the solution be orthogonal to the nullspace of $A^{T}$. The following proceedure may be used. Let $U \Sigma H^{T}=A$ be a full singular value decomposition of $A$. Let $z=U^{T} b$ and $y=H^{T} \theta$ so that $\Sigma y=z$. It follows that $y_{i}=z_{i} / \sigma_{i}$, $i=1, \ldots, n-1$, where $\sigma_{i}$ are the non-zero singular values of $A$. The last component $y_{n}$ is arbitrary since $\sigma_{n}=0$. The standard least squares solution can be obtained by setting $y_{n}=0$. To determine $y_{n}$ we require that $\sum_{i=1}^{n} \theta_{i}=0$. Since $\theta=H y$ we have

$$
\theta=\sum_{j=1}^{n-1} h_{i j} y_{j}+h_{i n} y_{n}
$$

Thus we require

$$
\sum_{i=1}^{n} \sum_{j=1}^{n-1} h_{i j} y_{j}+y_{n} \sum_{i=1}^{n} h_{i n}=0
$$

It follows that

$$
y_{n}=-\frac{\sum_{i=1}^{n} \sum_{j=1}^{n-1} h_{i j} y_{j}}{\sum_{i=1}^{n} h_{i n}} .
$$

The solution $\theta$ is then given by

$$
\theta=H y .
$$

The above numerical construction allows us to calculate the generic parameters $\lambda, V, D$ of the reduced Fokker-Planck equation (3.17) as functions of the various biophysical parameters of the tug-of-war model. These include the stall force $F_{s}$, the detachment force $F_{d}$, the maximum forward and backward velocities $v_{f}, v_{b}$, and the single motor binding/unbinding rates $\varepsilon_{0}, \pi_{0}$. For the sake of illustration, we consider two anterograde motors $\left(N_{q}=2\right)$ and one retrograde motor $\left(N_{r}=1\right)$, and plot $\lambda, V, D$ as functions of the stall force $F_{s}$ for various values of $v_{f}$, using parameter values 

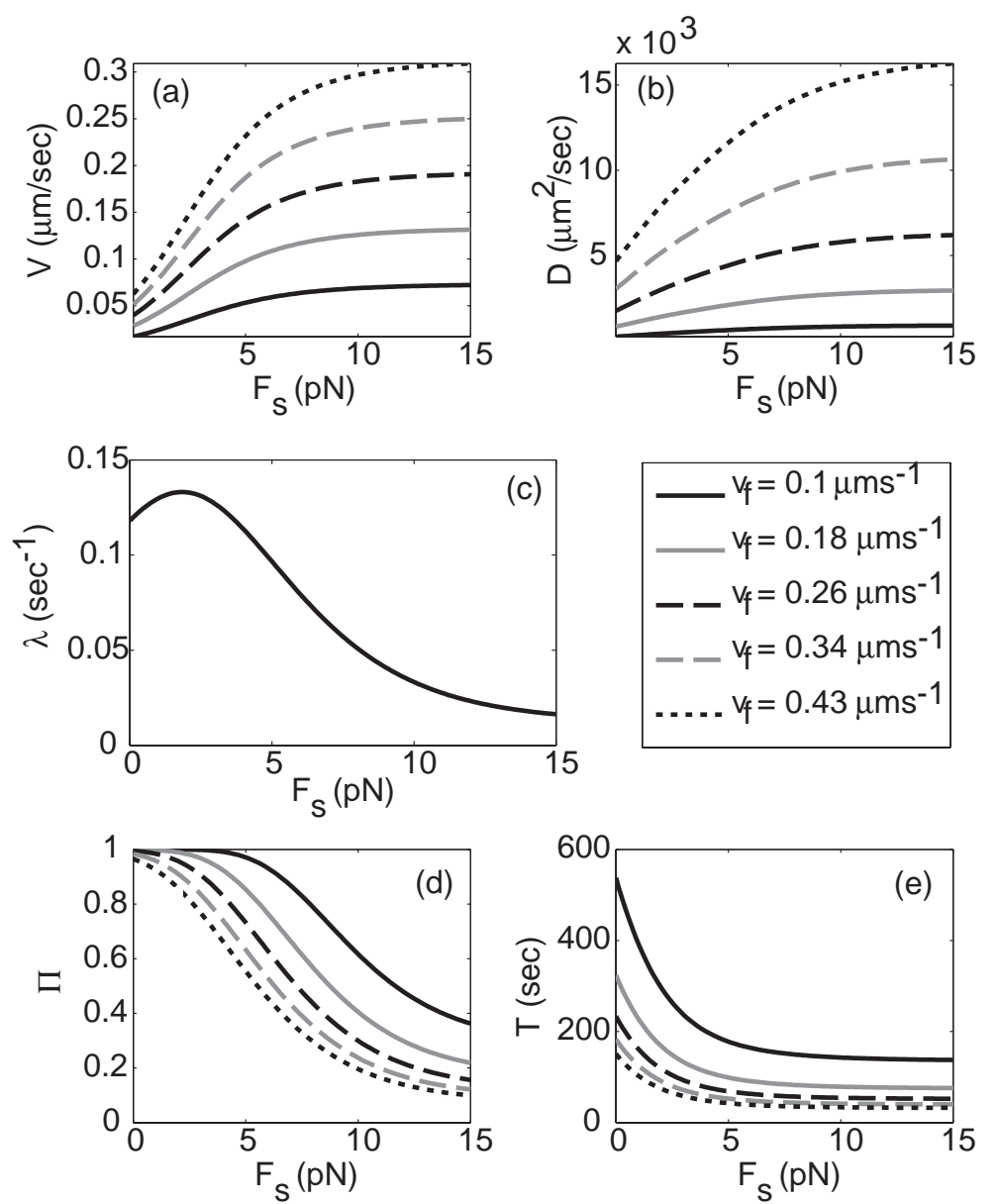

Fig. 7 Results of applying the quasi-steady state reduction to the tug-of-war model of directed intermmitent search. (a,b) Plot of the drift velocity $V$ and the diffusivity $D$ as functions of the single-motor stall force $F_{s}$ for various values of the maximum forward velocity $v_{f}$. (c) Plot of the detection rate $\lambda$ as a function of $F_{s}$. (d,e) Plot of the hitting probability $\Pi$ and the MFPT $T$ as functions of $F_{s}$ for various values of $v_{f}$. We use parameter values based on experimental data [31]: $k_{0}=0.5 \mathrm{sec}^{-1}, F_{d}=3 p N, \varepsilon_{0}=1 \mathrm{sec}^{-1}, \pi_{0}=5 \mathrm{sec}^{-1}$, and $v_{b}=0.006 \mu \mathrm{m} / \mathrm{sec}$.

extracted from experimental studies of kinesin [31]. The results are shown in Fig 7. It can be seen that both $V$ and $D$ are increasing functions of the stall force $F_{s}$, whilst the target detection rate $\lambda$ is maximized at small values of $F_{s}$. (We find that $\lambda$ is invariant with respect to changes in $v_{f}$ ). Combining these results with the plots of $\Pi$ and $T$ in Fig. 3, suggests that both the hitting probability and MFPT are decreasing functions of $F_{s}$, which is indeed found to be the case (see Fig. 7d,e). Hence, reducing the stall force increases the hitting probability but at the expense of increasing the MFPT. It should 


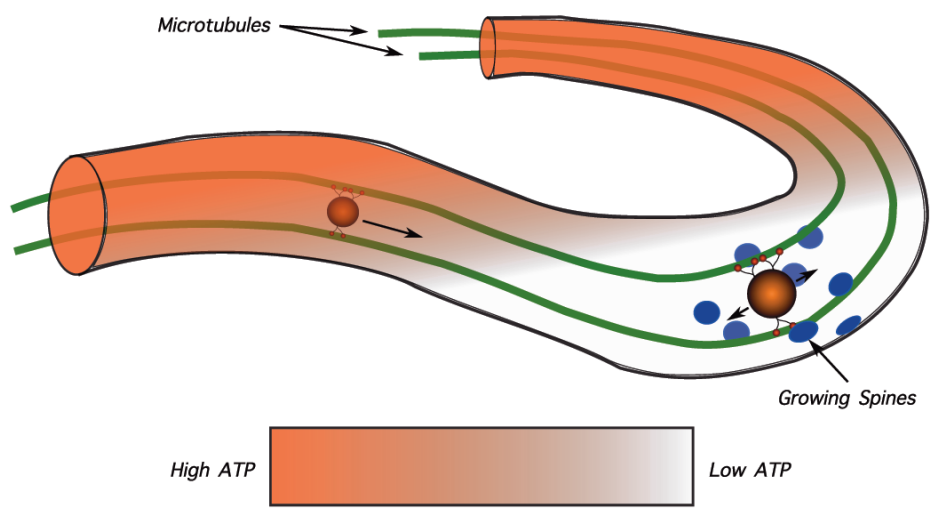

Fig. 8 Model of ATP dependent cargo transport. A motor driven cargo undergoes a transition from a directed transport state to a search state when encountering a region with low ATP concentration, such as a region of synapses undergoing activity-based morphological restructuring. In the search state the drift velocity and diffusivity is reduced and the detection rate is increased. The directed transport state is characterized by a large drift velocity and diffusivity.

be noted that the limit $F_{s} \rightarrow 0$ for fixed $v_{f} \neq 0$ is unphysical, since it would lead to infinite single motor speeds, see equation (5.1). Nevertheless, in this limit, the cargo force $F_{c} \rightarrow 0$ whereas the state velocities are left unchanged, see equations (5.7) and (5.9). This implies that the unbinding rate is given by the single motor rate $\varepsilon_{0}$ so that the motors no longer interact.

Our results regarding the dependence of the random search on the stall force suggest a possible signaling mechanism, whereby the level of metabolic activity can affect the behavior of motor driven transport in a manner that will impact where and when the cargo will be delivered. Recall that the kinesin motor must use energy from ATP hydrolysis to do work. (A detailed model of the kinesin motor and its dependence on the concentration of ATP can be found in [24]). Thus we expect the stall force $F_{s}$ and the forward velocity $v_{f}$ to be increasing functions of ATP concentration. Hence, in regions where ATP levels are low the corresponding reduction in the stall force $F_{s}$ implies that the particle is more likely to be in a search phase with small diffusion/drift and a high detection rate. Conversely, when ATP levels are high the particle has a higher probability of being in a non-search phase with larger drift/diffusion and a low detection rate. If the ATP concentration varies in space due to metabolic processes, such as activity-based changes in synaptic morphology thought to underly learning and memory[26], there 
will be a transition between search oriented dynamics at low ATP levels and movement oriented dynamics at high ATP levels (see Fig 8). This adaptive search scheme could provide a mechanism for generating a high hitting probability without a corresponding increase in the MFPT.

\section{Discussion}

In this paper we carried out a quasi-steady state reduction of a linear reaction-hyperbolic master equation describing the random intermittent search for a hidden target by a motor-driven particle moving on a onedimensional filament track. The resulting scalar Fokker-Planck equation was used to calculate the hitting probability and conditional mean first passage time (MFPT) for finding the target in terms of the effective drift velocity $V$, diffusivity $D$ and target absorption rate $\lambda$ of the random search. The quasi-state reduction provides a systematic method for expressing $V, D$ and $\lambda$ as functions of the various biophysical parameters of the corresponding motor transport model, and thus provides a general framework for characterizing the efficacy of the random search. In the particular case of a tugof-war model of multiple motors pulling a cargo [31], we determined how the hitting probability and MFPT depend on the stall force of the motors, and used this to suggest a mechanism whereby ATP concentration could function as a cargo localization signal.

One important extension of our work is to take into account the extensive branching structure of the dendritic tree. This can be handled by solving the Fokker-Planck equation on a tree, assuming continuity of the solution and conservation of flux at each branch point. As we show elsewhere [33], the presence of multiple branch points strongly reduces the efficacy of delivering cargo from the soma to distant synapses, suggesting that more local search strategies are necessary. This is consistent with the finding that clusters of immobile transport vesicles are found at branch nodes along the dendritic tree, which are subsequently recruited following activation of the cell [37]. A second extension concerns candidate signaling mechanisms for releasing cargo at synaptic sites. For example, it would be interesting to explore how variations in ATP concentration interact with the motor transport of mitochondria, which themselves regulate ATP concentration. For example, it has been found that active transport maintains a uniform distribution of mitochondria in axons such that mitochondria with a greater membrane potential undergo anterograde transport, whereas those with a low membrane potential undergo retrograde transport [29]. Other possible signaling molecules include microtubule associated proteins (MAPs) and calcium [17]. A third extension would be to develop a population-level model of the transport and delivery of cargo to multiple targets and the resulting competition for resources. Incorporating both global and local signaling mechanisms from synaptic targets would then allow us to explore the role of motor transport in synaptic plasticity $[8,34]$ and synaptogenesis [42], for 
example. Finally, it would be interesting to consider other factors that could constrain the "optimality" of the search process. One obvious example is metabolic cost, since it is likely that the fast ballistic state will be more energetically demanding than the slow search state. This is also likely to constrain how many motor-cargo complexes are produced during activation of a cell.

\section{Acknowledgements}

This publication was based on work supported in part by the National Science Foundation (DMS-0813677) and by Award No KUK-C1-013-4 made by King Abdullah University of Science and Technology (KAUST).

\section{References}

1. P. W. Baas, J. S. Deitch, M. M. Black and G. A. Banker. Polarity orientation of microtubules in hippocampal neurons: uniformity in the axon and nonuniformity in the dendrite. Proc. Natl. Acad. Sci. USA 85:8335-8339, 1988.

2. H. Bannai, T. Inoue, T. Nakayama, M. Hattori, and K. Mikoshiba, Kinesin dependent, rapid bidirectional transport of ER sub-compartment in dendrites of hippocampal neurons.. J Cell Sci, 117:163-175, 2004.

3. A. J. Bean (editor). Protein trafficking in neurons (Academic Press, MA 2007).

4. J. W. Bell, Searching Behaviour, The Behavioural Ecology of Finding Resources, London: Chapman and Hall, 1991.

5. O. Benichou, M. Coppey, M. Moreau, P. Suet, and R. Voituriez, Optimal search strategies for hidden targets. Phys. Rev. Lett., 94:198101, 2005.

6. O. Benichou, C. Loverdo, M. Moreau, and R. Voituriez, A minimal model of intermittent search in dimension two. J. Phys. A, 19: 065141, 2007.

7. O. G. Berg, R. B. Winter and P. H. von Hippel. Diffusion-driven mechanisms of protein translocation on nucleic acids. 1. Models and theory. Biochemistry 20:6929-6948, 1981.

8. C. R. Bramham and D. G. Wells. Dendritic mRNA: transport, translation and function. Nat. Rev. Neurosci. 8:776-789, 2007.

9. P. Bressloff and J. Newby, Directed intermittent search for hidden targets. New J. Phys., 11: 023033, 2009.

10. E. Brooks, Probabilistic methods for a linear reaction-hyperbolic system with constant coefficients. Ann. Appl. Prob., 9:719-731, 1999.

11. A. Brown. Slow axonal transport: stop and go traffic in the axon. Nat. Rev. Mol. Cell Biol. 1:153-156, 2000.

12. K. J. De Vos, A. J. Grierson, S. Ackerley, and C. C. J. Miller, Role of axonal transport in neurodegenerative diseases. Ann. Rev. of Neurosci. 31: 151-173, 2008.

13. J. Dynes and O. Steward, Dynamics of bidirectional transport of ARC MRNA in neuronal dendrites. J. Comp. Neurol., 500: 433-447, 2007.

14. A. Friedman and G. Craciun, Approximate traveling waves in linear reactionhyperbolic equations. Siam J. Math. Anal., 38:741-758, 2006. 
15. A. Friedman and B. Hu, Uniform convergence for approximate traveling waves in linear reaction-hyperbolic systems. Indiana Univ. Math. J., 56:2133-2158, 2007.

16. C. W Gardiner. Handbook of stochastic methods for physics, chemistry, and the natural sciences. Springer-Verlag, Berlin, 3rd edition, 2004.

17. A. Y. N. Goldstein, X. Wang, and T. L. Schwarz, Axonal transport and the delivery of pre-synaptic components. Curr. Opin. Neurobiol., 18:495-503, 2008.

18. S. E. Halford and J. F. Marko. How do site-specific DNA-binding proteins find their targets? Nucl. Acid Res. 32:3040-3052, 2004.

19. N. Hirokawa and R. Takemura, Molecular motors and mechanisms of directional transport in neurons. Nat. Rev. Neurosci. 6:201-214, 2005.

20. J. Howard. Mechanics of motor proteins and the cytoskeleton. Sianuer, MA 2001.

21. R. L. Kelleher, A. Govindarajan and S. Tonegawa. Translational regulatory mechanisms review in persistent forms of synaptic plasticity Neuron 44:59-73, 2004.

22. M. J. Kennedy and M. D. Ehlers. Organelles and trafficking machinery for postsynaptic plasticity. Ann. Rev. Neurosci. 29:2325-2362, 2006.

23. C. Kural, H. Ki, SD. Syed, G. Goshima, V. I. Gelfand and P. R. Selvin. Kinesin and Dynein move a peroxisome in vivo: A tug-of-war or coordinated movement. Science 308:1469-1472, 2005.

24. S. Liepelt and R. Lipowsky, Kinesin's network of chemomechanical motor cycles.. Phys. Rev. Lett, 98:258102, 2007.

25. R. Knowles, J. Sabry, M. Martone, T. Deerinck, M. Ellisman, G. Bassell, and K. Kosik, Translocation of RNA granules in living neurons. J. Neurosci. 16:7812-7820, 1996.

26. R. Lamprecht and J. LeDoux. Structural plasticity and memory. NAT. Rev. Neurosci. 5:45-54, 2004.

27. C. Loverdo, O. Benichou, M. Moreau, and R. Voituriez, Enhanced reaction kinetics in biological cells. Nat. Phys. 4:134-137, 2008.

28. M. P. Mattson, M. Gleichmann, and A. Cheng, Mitochondria in neuroplasticity and neurological disorders. Neuron, 60: 748-766, 2008.

29. K. E. Miller and M. P. Sheetz. Axonal mitochondrial transport and potential are correlated. J. Cell Sci. 117:2791-2804, 2004.

30. R. L. Morris and P. J. Hollenbeck. The regulation of bidirectional mitochondrial transport is coordinated with axonal outgrowth. J. Cell Sci. 104:917-927, 1993.

31. M. J. I. Mueller, S. Klumpp, and R. Lipowsky, Tug-of-war as a cooperative mechanism for bidirectional cargo transport by molecular motors. Proc. Nat. Acad. Sci. USA, 105:4609-4614, 2008.

32. T. Nakata, S. Terada and N. Hirokawa. Visualization of the dynamics of synaptic vesicle and plasma membrane proteins in living axons. J. Cell Biol. 160: 659-674, 1998.

33. J. Newby and P. C. Bressloff. Directed intermittent search for a hidden target on a dendritic tree. Phys. Rev. E 80:021913, 2009.

34. S. V. Puthasnveettil, F. J. Monje, M. C. Miniaci, Y-B Choi, K. A. Karl, E. Khandros, M. A. Gawinowicz, M. P. Sheetz and E. R. Kandel. A new component in synaptic plasticity: upregulation of kinesin in the neurons of the gill-withdrwal reflex. Cell 135:960-973, 2008.

35. S. Redner, A Guide to First Passage Time Processes. Cambridge: Cambridge University Press, 2001. 
36. M. C. Reed, S. Venakides, and J. J. Blum, Approximate traveling waves in linear reaction-hyperbolic equations. SIAM J. Appl. Math. 50: 167-180, 1990.

37. M. S. Rook, M. Lu, and K. S. Kosik, CaMKIIL 3' untranslated regions-directed MRNA translocation in living neurons: Visualization by GFP linkage. J. Neurosci., 20: 6385-6393, 2000.

38. G. B. Stokin and L. S. B. Goldstein. Axonal transport and Alzheimer's disease. Ann. Rev. Biochem. 75:607-627, 2006.

39. M. A. Sutton and E. M. Schuman. Dendritic protein synthesis, synaptic plasticity, and memory. Cell 127: 49-58, 2006.

40. R. D. Vale, The molecular motor toolbox for intracellular transport. Cell, 112: 467-480, 2003.

41. G. M. Viswanathan, S. V. Buldyrev, S. Havlin, M. G. E. da Luz, E. P. Raposo and H. E. Stanley. Optimizing the success of random searches. Nature, 401:911-914, 1999

42. C. Waites, A. Craig, and C. Garner, Mechanisms of vertebrate synaptogenesis, Ann. Rev. Neurosci. 28:251-274, 2005.

43. P. Washbourne, X-B Liu, E. G. Jones and A. K. McAllister, Cycling of NMDA receptors during trafficking in neurons before synapse formation, J. Neurosci. 24:8253-8264, 2004.

44. M. A. Welte. Bidirectional transport along microtubules, Curr. Biol. 14: 525, 2004. 


\section{RECENT REPORTS}

04/09 Stability analysis of reaction-diffusion systems with timem- Madzvamuse dependent coefficients on growing domains

Gaffney

Maini

05/09 Onsager reciprocity in premelting solids

Peppin

Spannuth

Wettlaufer

06/09 Inherent noise can facilitate coherence in collective swarm motion

Yates et al.

07/09 Solving the Coupled System Improves Computational Efficiency

Southern of the Bidomain Equations

Plank

Vigmond

Whiteley

08/09 Model reduction using a posteriori analysis

Whiteley

09/09 Equilibrium Order Parameters of Liquid Crystals in the LaudauDe Gennes Theory

10/09 Landau-De Gennes theory of nematic liquid crystals: the OseenFrank limit and beyond

Majumdar

Zarnescu

11/09 A Comparison of Numerical Methods used for Finite Element Modelling of Soft Tissue Deformation

Pathmanathan

Gavaghan

Whiteley

12/09 From Individual to Collective Behaviour of Unicellular Organisms: Recent Results and Open Problems

Xue

Othmer

Erban

13/09 Stochastic modelling of reaction-diffusion processes: algorithms for bimolecular reactions

Erban

Chapman

14/09 Chaste: a test-driven approach to software development for physiological modelling

15/09 Block triangular preconditioners for PDE constrained optimization

Pitt-Francis et al.

Rees

Stoll

16/09 From microscopic to macroscopic descriptions of cell migration on Baker growing domains

Yates

Erban

17/09 The Influence of Gene Expression Time Delays on Gierer- Seirin Lee Meinhardt Pattern Formation Systems

Gaffney

Monk 
18/09 Analysis of a stochastic chemical system close to a sniper bifurcation of its mean field model

Erban et al.

19/09 On the existence and the applications of modified equations for stochastic differential equations

20/09 Pebble bed: reflector treatment and pressure velocity coupling

Charpin et al.

21/09 A finite difference method for free boundary problems

Fornberg

22/09 Tangent unit-vector fields: nonabelian homotopy invariants and the Dirichlet energy

Majumdar

Robbins

Zyskin

23/09 Morphological instability of a nonequilibrium icecolloid interface

Peppin

Majumdar

Wettlaufer

24/09 The effect of polar lipids on tear film dynamics

Aydemir

Breward

Witelski

25/09 Preconditioning for active set and projected gradient methods as

Stoll semi-smooth Newton methods for PDE-constrained optimization

Wathen with control constraints

26/09 Functional differential equations arising in cell-growth

Wake

Begg

27/09 A Cell Growth Model Revisited

Derfel

van Brunt

Wake

Copies of these, and any other OCCAM reports can be obtained from:

Oxford Centre for Collaborative Applied Mathematics Mathematical Institute

24 - 29 St Giles'

Oxford

OX1 3LB

England

www.maths.ox.ac.uk/occam 